\title{
Structural investigation and compression of a co-crystal of indomethacin and saccharin.
}

Received 00th January 20xx, Accepted 00th January 20xx DOI: $10.1039 / \times 0 \times x 00000 x$

\author{
Lauren E. Connor, ${ }^{\text {ab }}$ Antony D. Vassileiou, ${ }^{d}$ Gavin W. Halbert, ${ }^{\mathrm{a}, \mathrm{c}}$ Blair F. Johnston ${ }^{\mathrm{a}, \mathrm{c}-\mathrm{e}}$ and lain D.H. \\ Oswald*a
}

The co-crystalline structure of the non-steroidal, anti-inflammatory indomethacin with the non-toxic, Generally Regarded As Safe (GRAS) sweetener component saccharin was investigated up to $6.33 \mathrm{GPa}$ using a Diamond Anvil Cell (DAC). Single crystal X-ray diffraction measurements show that the cocrystal remains in the same triclinic, $\mathrm{P}-1$, phase throughout the compression with a significant reduction in void space (155.69 to $\left.55.61 \AA^{3}\right)$. Information on the response of different types of intermolecular interactions to external force at the same time is enabled by the use of a co-crystal. We have rationalised that the length and compression rate of the saccharin amide dimer in the cocrystal is caused by the dimer sitting in a 'pocket' surrounded by the indomethacin framework. This framework reduces the effects of molecular packing on the dimer allowing for an ideal hydrogen bonding geometry.

Keywords: Co-crystal; high pressure; multicomponent system; indomethacin.

\section{Introduction}

Co-crystallisation is a branch of solid state modification that has been of growing interest in the energetic material, ferroelectric and pharmaceutical industry in recent years. ${ }^{1-6}$ Much akin to its fellow branch of solid state modification, salt formation, cocrystallisation is the incorporation of two or more species in the crystalline structure. Co-crystallisation has a much greater scope due to the use of neutral coformers and the lack of requirement for an ionisable group. ${ }^{7}$ Crystal engineering modifications are sought to explore a few avenues of interest but one common interest spanning across all industries is the alteration of the physicochemical properties. ${ }^{8,9} \mathrm{~A}$ change in the physicochemical properties could also mean a change in the mechanochemical properties and functionality of the singular component material on the larger, potentially tangible, product scale. Interesting macroscopic mechanical properties have been observed in co-crystals of vanillin, ${ }^{10}$ caffeine, ${ }^{11}$ and chlorophenol derivatives ${ }^{12}$ where extreme flexibility in crystals

\footnotetext{
a. Strathclyde Institute of Pharmacy and Biomedical Sciences, University of Strathclyde, 161 Cathedral Street, Glasgow, G4 ORE, U.K.

b. Collaborative International Research Programme: University of Strathclyde and Nanyang Technological University, Singapore, Technology Innovation Centre, 99 George Street, Glasgow, G1 1RD, U.K.

c. EPSRC Centre for Innovative Manufacturing in Continuous Manufacturing and Crystallisation, University of Strathclyde, Technology Innovation Centre, 99 George Street, Glasgow, G1 1RD, U.K.

d. EPSRC ARTICULAR, University of Strathclyde, Technology Innovation Centre, 99 George Street, Glasgow, G1 1RD, U.K.

e. National Physical Laboratory, Hampton Road, Teddington, Middlesex, TW11 OLW Electronic Supplementary Information (ESI) available: Crystallographic Information for the INSA structures at pressure, hydrogen-bonding parameters for each structure See DOI: 10.1039/x0xx00000x
}

have been observed. These co-crystals can be physically bent (exhibiting plastic deformation) due to the anisotropy of the interactions between molecules where strong and weak interactions are present perpendicular to each other. These materials still show a crystalline nature even after deformation. ${ }^{11,13}$ To fundamental scientists, co-crystallisation offers something of value on the molecular scale; the potential to investigate intermolecular interactions which may not have occurred within the structure of a singular component. By adding another component to the structure there are new synthons to analyse which open up a wealth of new molecular opportunities. In understanding these competing interactions we can begin to understand how the molecules self-assemble into the co-crystalline form. Co-crystals may contain components that have a large difference in the number of atoms hence the balance of contribution of different intermolecular interactions in these systems can be quite different to pure systems. Whilst in many systems hydrogen bonding interactions are the linkage of the two components in the crystal structure, weaker dispersive forces can play a significant role in the overall energy of the structure. By understanding the basic fundamentals of the structure, the properties can be explained and possibly controlled but even when we have understood a system invariably polymorphism arises. A recent study by Cruz-Cabeza et al. ${ }^{14}$ has shown through database mining that polymorphism in co-crystalline phases are equally as common as with pure components. The initial concept of co-crystallisation was to try to inhibit or reduce the propensity for polymorphism but this has proved to be not the case. ${ }^{15-17}$ Considering this realisation, we have set about to use high pressure to evaluate effects of pressure on co-crystalline systems. Using pressure we can: i) survey the thermodynamic stability of ambient pressure forms; ${ }^{18-21}$ ii) access to metastable 
polymorphs; ${ }^{22-24}$ and iii) investigate the response of hydrogen bonds with respect to pressure. ${ }^{25-31}$

Indomethacin is a Non-Steroidal Anti-Inflammatory (NSAID) that is known for its ability to reduce fever, pain, stiffness and swelling and can be prescribed for migraines. There are 10 different multi-component forms found in the Cambridge Structural Database (CSD). ${ }^{32}$ In its pure form, Indomethacin has been well-characterised under ambient and high-pressure conditions. Under ambient conditions there are three polymorphs $\alpha-\gamma$; a fourth $\delta$-form is a hydrate. At ambient pressure, the $\gamma$-form is the most stable form which was demonstrated by Okumura et al. where they slurried the $\alpha$-form of indomethacin in ethanol over the course of four days and observed that transformed to the $\gamma$-form. At high pressure, however, the behaviour of the slurry changes. At a modest pressure of $0.4 \mathrm{GPa}$, the transformation of the $\alpha$-form to the $\gamma$ form is reversed indicating that whilst metastable at ambient pressure, the $\alpha$-form is more stable at higher pressures. ${ }^{33} \mathrm{~A}$ compression of the $\gamma$-form to $0.4 \mathrm{GPa}$ without ethanol solvent did not reveal any polymorphic transformation which reveals the significance of the solvent in mediating these pressureinduced phase transitions. ${ }^{34}$

Given the rich behaviour of Indomethacin, we have chosen to investigate the co-crystal of indomethacin and saccharin (INSA) under high-pressure conditions. It is a well-characterised system known to have altered physicochemical properties compared with indomethacin itself such as a higher solubility and bioavailability. ${ }^{35,36}$ In the previous studies, there is no indication of any polymorphism. In the co-crystal, the indomethacin and saccharin molecules hydrogen bond with themselves allowing both types of hydrogen bond (carboxylic acid dimer and amide dimer) to be assessed under a range of pressure conditions.

\section{Experimental}

\section{General procedure}

A Merrill-Bassett diamond-anvil cell (DAC) with an opening angle of $80^{\circ}$ was used for the experiments. The opposing gemquality diamonds with a culet size of $600 \mu \mathrm{m}$ were used with Boehler-Almax tungsten carbide backing seats. The DAC sample chamber was a $300 \mu \mathrm{m}$ diameter hole drilled into a tungsten $10 \mathrm{~mm} \times 10 \mathrm{~mm}$ square gasket pre-indented to a thickness of $90 \mu \mathrm{m}$ (Almax Easylab $\mu$ Driller). A ruby sphere of approximately $15 \mu \mathrm{m}$ diameter was loaded into the chamber along with the sample to measure the pressure in situ via the ruby fluorescence technique. ${ }^{37} \mathrm{~A}$ Horiba XplorA Raman spectrometer equipped with a $532 \mathrm{~nm}$ laser was used to measure the ruby fluorescence.

\section{Co-crystal formation}

A 1:1 stoichiometric co-crystal of indomethacin and saccharin (INSA) was grown from ethyl acetate via a small batch evaporative co-crystallisation $(35.7 \mathrm{mg}$ of indomethacin to $18.32 \mathrm{mg}$ of saccharin in $1 \mathrm{ml}$ ). A crystal of dimensions $0.5 \mathrm{~mm} \mathrm{x}$
$0.3 \mathrm{~mm} \times 0.25 \mathrm{~mm}$ was used to determine the crystal structure at ambient pressure.

\section{Diamond anvil cell preparation}

For the compression study a prismatic crystal of the co-crystal was loaded into the DAC with petroleum ether (40:60) acting as the pressure-transmitting medium to allow for hydrostatic compression; the initial sealing of the cell introduced a pressure of $0.55 \mathrm{GPa}$. Single crystal X-ray diffraction data were collected with the DAC in a single orientation throughout the study, attempts to improve on the data completeness by collecting a second orientation and merging the data with the initial dataset failed to provide a significant improvement in the completeness or structure refinement. Due to a pressure jump from 0.55GPa to $3.33 \mathrm{GPa}$, another cell was loaded in the same manner with another crystal. A dataset was taken at $1.66 \mathrm{GPa}$ to fill the gap in the compression study.

\section{Single-crystal X-Ray diffraction}

\section{Ambient crystal collection, processing and refinement}

$X$-ray diffraction intensities were collected from a sequence of six scans at 293K using a Bruker APEX II CCD diffractometer with an Incotec I $\mu \mathrm{S}$ microsource (Mo $K \alpha$ radiation, $\lambda=0.71073 \AA$ ) . Data were indexed and then integrated using SAINT as incorporated in the APEX II software. ${ }^{38}$ An absorption correction was applied using SADABS. ${ }^{39}$ The crystal structure at ambient pressure was refined within OLEX $2^{40}\left(F^{2}\right)$ using the atomic coordinates from the Cambridge Structural Database (CSD refcode UFERED). All heavy atoms were anisotropically refined. Hydrogen atoms were placed in calculated positions and constrained to ride their parent atom as per procedure in OLEX2. The crystallographic data can be found in Table S1.

\section{Compression study collection, processing and refinement}

The high-pressure data were collected and treated in a similar manner to the ambient procedure described above. $X$ ray diffraction intensities were collected from a sequence of twelve scans at $293 \mathrm{~K}$ following the procedure described by Dawson et al. Data were indexed and then integrated using SAINT in conjunction with dynamic masks. ${ }^{41}$ An absorption correction was applied in two stages using both SHADE and SADABS, respectively. ${ }^{39,42}$ Known coordinates from the ambient pressure model were used as a starting model for the refinement. For high-pressure datasets the heavier atoms (S-35 and $\mathrm{Cl}-1$, see $\mathrm{Fig} 1$ ) were refined anisotropically and the remainder of the heavy atoms were refined isotropically due to the limited data and large number of atoms. Additionally, distance restraints were applied to the high pressure structures of INSA using distances taken from a Mogul geometry search of the CSD as implemented in Materials Mercury. ${ }^{43}$ The geometry of the phenyl rings were constrained. Hydrogen atoms were placed in calculated positions and constrained to ride their parent atoms. The refined model from each pressure point was used as a starting model for each subsequent model. The crystallographic data can be found in Table S1.

H-bonding length distribution in Cambridge Structural Database 
The CSD version 5.39 (March $2018+3$ updates until May 2018) was used along with Conquest Version 1.21 to search for $\mathrm{H}$ bond distributions. ${ }^{32}$ The first search for crystal structures with an intermolecular (carboxylic acid) $\mathrm{O}-\mathrm{H} \cdots \mathrm{O}$ dimer contact length between $2.45 \AA$ and $2.75 \AA$ produced 4977 refcodes. The second search for crystal structures with an intermolecular (amide) $\mathrm{N}$ $\mathrm{H} \cdots \mathrm{O}$ dimer contact length between $2.65 \AA$ and $3.1 \AA$ produced 7600 refcodes. Both searches excluded all errors, polymeric, ion and powder structures and considered only organic molecules with 3D coordinates determined. An additional constraint of no alkali metals was used since the software's native definition of an organic molecule includes these species.

Using CSD Python API, a restricted CSD entry list of compounds under high-pressure conditions was produced (2454 CSD refcodes). Only refcodes with pressure explicitly stated in the experimental conditions were taken into account. The exclusion parameters previously described were also applied here for consistency with the original search. The restriction narrowed the results of the carboxylic acid dimer search to 45 refcodes, equating to 12 compounds, and the amide dimer search to 30 refcodes, equating to 5 compounds.

\section{Dimer potential energy calculations}

The coordinates of the saccharin dimer were taken from the ambient pressure structure of saccharin (CSD refcode SCCHRN06). The dimer was extracted from the crystal structure using Avogadro ${ }^{44}$ and 19 different D...A distances were formulated for input into Gaussian $09^{45}$ using Pixelc. ${ }^{46-48} \mathrm{~A}$ basisset of MP2/6.31G** was chosen to calculate the dimer energy and the lowest energy was set to zero.

\section{Results and Discussion}

\section{Structure}

The crystals grown from ethyl acetate were of similar size and consistent in crystal habit indicating that the batch were all the same material. The unit cell information was consistent with those found in the Cambridge Structural Database (CSD) for the 1:1 stoichiometric co-crystal of INSA (CSD refcode: UFERED)
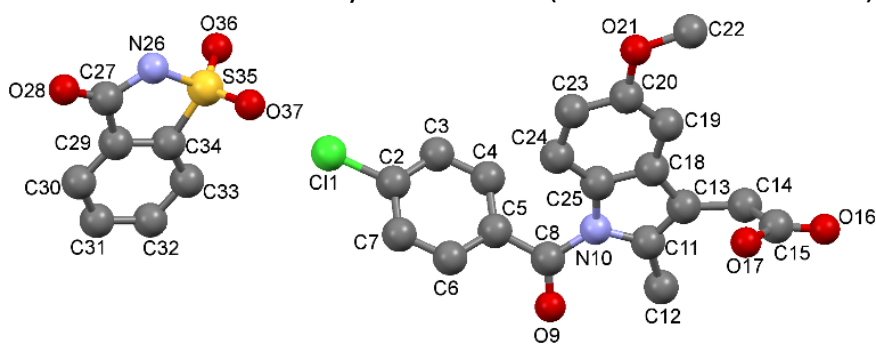

(Fig. 1). ${ }^{32,35}$ INSA crystallises in space group P-1 with one molecule of each component in the asymmetric unit.

Figure 1: The asymmetric unit of Indomethacin(right):saccharin(left) co-crystal with numbering scheme (hydrogen atoms omitted for clarity). The colours of the elements are consistent throughout paper unless otherwise stated. (carbon, grey; oxygen, red; sulphur, yellow; nitrogen, blue; chlorine, green; hydrogen, white,
shown in Fig. 2)

Indomethacin is an indole derivative with a chlorobenzoyl ring, methoxy group and carboxylic acid presenting opportunities for hydrogen bonding with suitable donors. Saccharin, the artificial sweetener, is a dioxobenzothiazol and is considerably smaller than indomethacin. The crystal structure of INSA consists of dimers of each component linked through close interaction between the $\mathrm{NH}$ of saccharin and $\mathrm{C}=\mathrm{O}$ of the indomethacin. Utilising this close interaction, the two components form a chain along the $b$-axis as shown in Fig. 2. Neighbouring chains run parallel to each other interlocking through non-directional $\mathrm{CH}-\pi$ interactions between the chlorobenzoyl rings (Fig. 3).

Pixel calculations ${ }^{47,49}$ of the INSA co-crystal, reveal that the strongest molecule to molecule interactions are those associated with the hydrogen bonded dimers; the carboxylic acid dimer between indomethacin molecules (total $83.6 \mathrm{~kJ} \mathrm{~mol}^{-1}$; O16 ${ }^{\prime} 017^{\prime} 2.677(3) \AA$ ) and the sulphonamide dimer between the saccharin components (total $60.2 \mathrm{kJmol}^{-1}$; N26“. O28' 2.884(3) A). The third and fourth notable interaction can be attributed to a $\pi / \pi$ interaction (indomethacin to saccharin, total $-50.3 \mathrm{kJmol}^{-1}$; dispersion $-51.1 \mathrm{kJmol}^{-1}$ ) and $\mathrm{a} \mathrm{CH} / \pi$ interaction (indomethacin to indomethacin, total $-46.1 \mathrm{kJmol}^{-1}$; dispersion $53.6 \mathrm{kJmol}^{-1}$ ) respectively. These latter two molecule-molecule interactions are surprisingly strong given that they are predominantly comprised of dispersive forces. The bicyclic

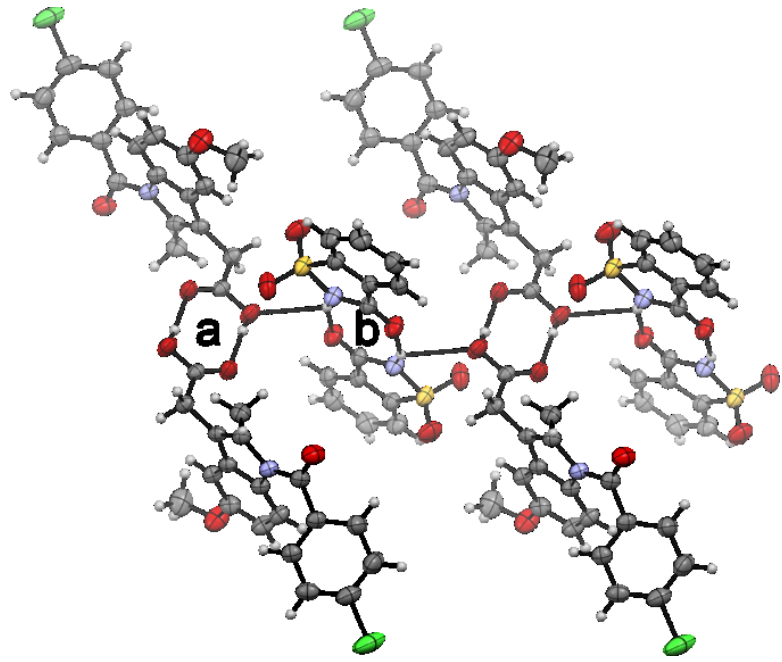

Figure 2: A section of the structure of indomethacin and saccharin with hydrogen bonding contacts shown. Depicted $\mathbf{a}$ and $\mathbf{b}$ indicate a strong carboxylic acid dimer formed by indomethacin molecules and the dimer between saccharin molecules, respectively.

moieties of the indomethacin and saccharin are well-aligned which contributes significantly to the attractive dispersive forces. In this instance, the total energy of the interaction is nearing the typical energies for hydrogen-bonded interactions. The bicyclic indole moiety of the indomethacin interacts through a $\mathrm{CH}^{\cdots} \pi$ interaction with a neighbouring indomethacin molecules where the chlorobenzoyl of the second indomethacin is in an edge-to-face arrangement with the symmetry equivalent molecule $(-x,-y,-z)$. 


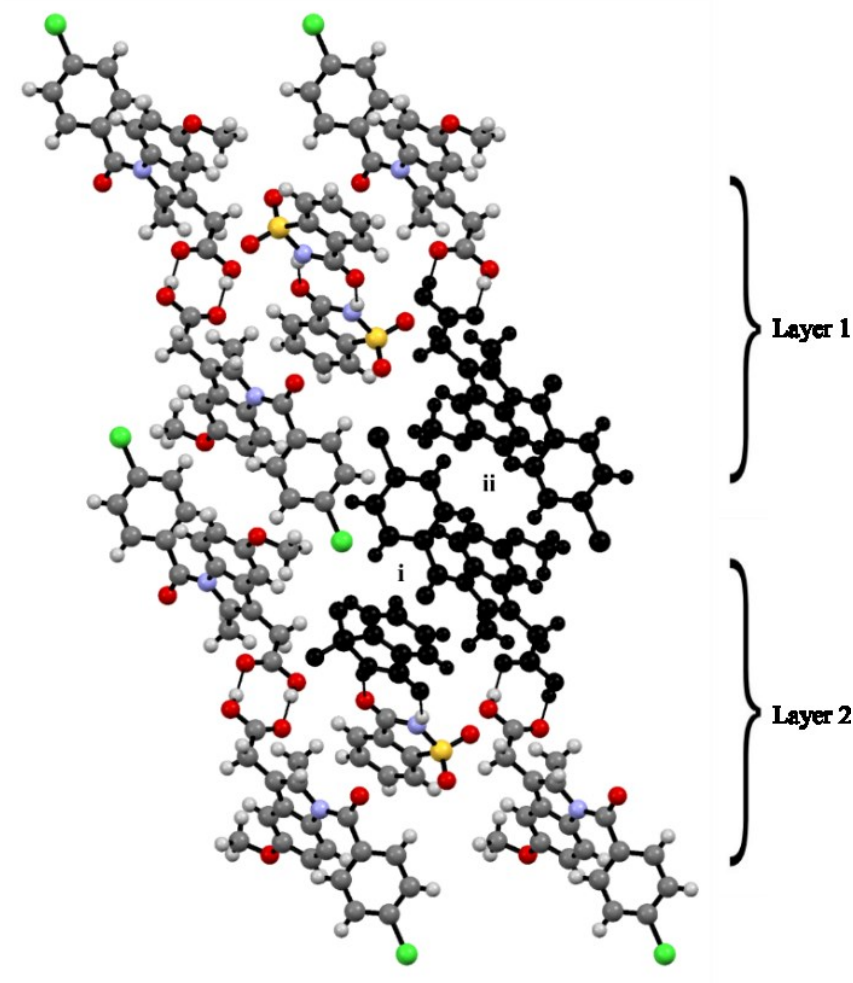

Figure 3: Section of two hydrogen bonded chains running parallel along the $b$-axis in the $a b$ plane interlocking through a $\pi / \pi$ interaction between indomethacin and in the blackened molecules.

\section{Compression study}

The co-crystal undergoes a $20 \%$ decrease in volume from $1210.1 \AA^{3}$ at ambient pressure to $974.5 \AA ̊$ at $5.06 \mathrm{GPa}$ (Figure 4). The $3^{\text {rd }}$ order Birch-Murnaghan Equation of State gives a bulk modulus ( $\left.\mathrm{K}_{0}\right)$ of $15(3) \mathrm{GPa}$ with a $\mathrm{V}_{0}=1199(12) \AA^{3}$ and $\mathrm{K}^{\prime}=$ 4.6(12). A further dataset taken at $6.33 \mathrm{GPa}$ was of such poor quality that no structural information could be derived even after collecting data in a different orientation, however, the unit cell information was used in the determination of the bulk modulus. The decrease in the quality of the crystal is attributed to reaching the limit of the hydrostatic medium.
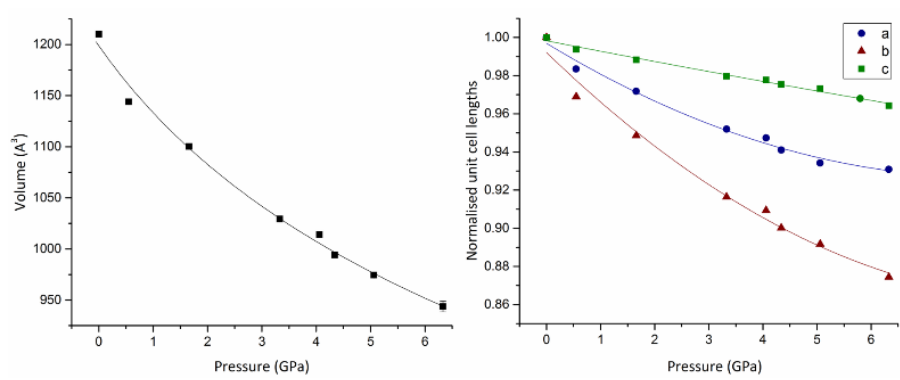

Figure 4: a) Unit cell volume of INSA as a function of pressure. The $3^{\text {rd }}$ order BirchMurnaghan Equation of State $\left(\mathrm{K}_{0}=15.46(13) \mathrm{GPa}, \mathrm{V}_{0}=1199.35\right.$ (4) $\AA^{3}, \mathrm{~K}^{\prime}=4.49$ (2)) is plotted alongside the data (line) (EoSfit 7$)^{50}$, ( $(b)$ Normalised unit cell lengths
as a function of pressure. The errors on each of the measurements is smaller than as a function of pressure. The errors on each of the measurements is smaller than
the symbol.

The unit cell axes display a smooth compression with decreases of $6.5 \%, 10.8 \%$ and $2.7 \%$ in the $a-, b$ - and $c$-axis respectively (Fig.
4). The large decrease in the $b$-axis is explained by the analysis of the void space in the structure and the absence of strong interactions between the indomethacin and saccharin dimers. Figure 5 shows the voids present in the structure at ambient pressure with large pockets of space between the chlorobenzoyl rings of neighbouring indomethacin molecules translated along the same chain. Over the course of the compression, these voids decrease from $17.8 \%$ of the unit cell to $4.7 \%$ at $5.06 \mathrm{GPa}$ (Fig. 5; Table 1). Even at the highest pressure of $6.33 \mathrm{GPa}$, the unit cell parameters that could be observed from the deteriorating diffraction pattern indicated the retention of this phase hence the void space will have compressed even further. Using PASCal ${ }^{51}$ the greatest direction of compression has a large component of the $b$-axis $[0.4317$ $0.8653-0.2546$ ] but the overall direction of this greatest strain is in the direction of the $\pi / \pi$ and $\mathrm{CH} / \pi$ interactions described earlier. The other two axes of strain are perpendicular to this and approximately perpendicular to each other. It is apparent that the voids are present in all three dimensions in the crystal structure and are partly accountable for the resistance to any phase transformation in the system i.e. in every direction compression can take place without necessary rearrangement of atoms to relieve repulsion between atoms. The small pocket of void space, in Fig. $5 d$, is 'protected' from compression to some extent due to the size and bulkiness of the indomethacin molecule with any reduction in the pocket necessitating compression of molecular groups substantially inside the sum of the van der Waals radii.

One of the significant developments over the compression is the change in the interaction energy between the indomethacin molecule and the saccharin molecule $(x-1,1+y$, $1+z)$. The close contact, and only real interaction between the molecules, is between the hydroxyl oxygen and carbonyl oxygen atoms indomethacin and saccharin respectively. The interaction between these molecules becomes highly repulsive increasing from $1.2 \mathrm{kJmol}^{-1}$ at ambient pressure to $7.2 \mathrm{kJmol}^{-1}$ at $5.06 \mathrm{GPa}$. There are very few attractive forces between the molecules hence the repulsion term dominates the molecular interaction.

One of the key aspects to this study was to investigate how intermolecular interactions change as a function of pressure. There have been a large number of studies of materials under pressure but the types of hydrogen bond that have been probed is not particularly diverse nor have there been a significant number of studies of particular hydrogen bonds except for, perhaps, ammonium-carboxylate interaction, due to studies of amino acids. ${ }^{29,52-58}$ INSA exists as a co-crystal with only homomeric hydrogen bonding interactions (carboxylic acid and amide dimers). We probed the CSD for similar hydrogen bonding interactions to those in our system. Analysis of hydrogen bonding distributions of similar dimer interactions are presented in Fig. 6. Due to the limited data from the highpressure experiment, the hydrogen bonding distances are

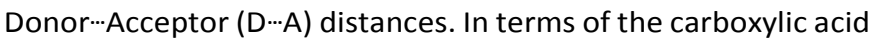
dimer, the mean distance is $2.643 \AA$. At ambient pressure, carboxylic acid dimer of INSA $(2.6803(18) \AA)$ is slightly above this value, however on compression to $5.06 \mathrm{GPa}$ the dimer reduces 
by $7 \%$ to $2.496(12) \AA$, below those observed in the database (Table S2).

Table 1: Percentage of the unit cell occupied by void space at each pressure. The probe radius was set to $0.5 \AA ̊$ with 0.1 grid spacing.

\begin{tabular}{|c|c|}
\hline Pressure (GPa) & Percentage of unit cell (\%) \\
\hline 0.0001 & 17.8 \\
\hline 0.55 & 12.7 \\
\hline 1.66 & 11.3 \\
\hline 3.33 & 6.9 \\
\hline 4.06 & 6.6 \\
\hline 4.34 & 5.5 \\
\hline 5.06 & 4.7 \\
\hline
\end{tabular}

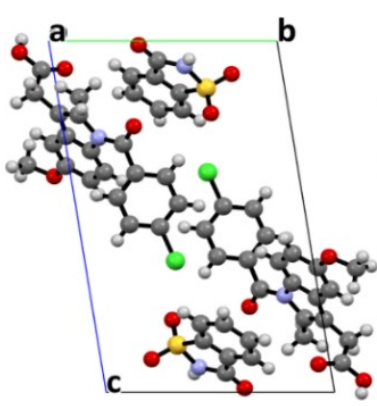

(a)

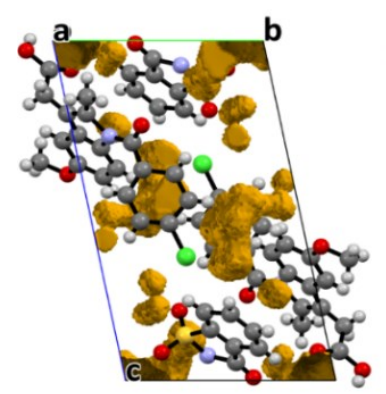

(c)

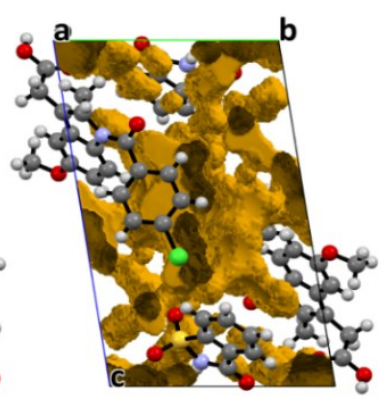

(b)

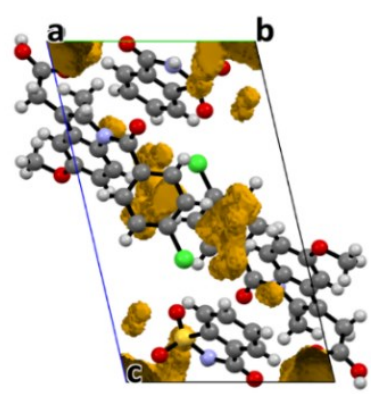

(d)
Figure 5: The structure of the unit cell down the $a$-axis (a). The void space at 0.0001 $\mathrm{GPa}(\mathrm{b}), 3.33 \mathrm{GPa}(\mathrm{c})$, and $5.06 \mathrm{GPa}(\mathrm{d})$ obtained by using probe radius of $0.5 \AA$ with
high definition of $0.1 \mathrm{~A}$.

The majority of the database structures are at ambient pressure but the lower end of the distribution has been used in previous work to show that a phase transition may be imminent during compression studies. ${ }^{59}$ Perhaps a more instructive comparison would be with other pressure studies to see if, despite changes to molecular connectivity, the hydrogen bonds are compressed to a similar level. Restricting the database to those refcodes that have pressure explicitly stated in their experimental conditions, there are 2454 refcodes reported in the CSD. 45 of these refcodes contain a carboxylic acid dimer contact. R,S-Ibuprofen ${ }^{60}$ and acetylenedicarboxylic acid ${ }^{61}$ are the only observations of a carboxylic acid dimer compression study in the database, via single crystal diffraction, that extends to the pressures that have been investigated here. In general, the other entries are mostly individual collections at particular pressures hence do not give information on how hydrogen bonds change with respect to pressure. Notable studies are $2+2$ cycloaddition reactions of 3-(2,5-difluorophenyl)prop-2-enoic acid (HESREF) ${ }^{62}$ and 3 -(2,6-difluorophenyl)acrylic acid ${ }^{63}$ under high pressure conditions that have been studied to 0.9 and 2.1 GPa respectively. The hydrogen bonded dimer in ibuprofen compresses to a similar value to our INSA example and at $4 \mathrm{GPa}$ it is observed at $2.549 \AA$ (cf. 2.529(10) $\AA$ for INSA at 4.06 GPa)(Table 3). Further compression of INSA to 5.06 GPa reduces the hydrogen bond further to $2.496(12) \AA$ to be amongst the shortest reported in the database. In the case of acetylenedicarboxylic acid the hydrogen bond is disordered but the 0 ...O distance reduces form $2.691 \AA$ to $2.557 \AA$ at $4.85 \mathrm{GPa}$ before undergoing a phase transformation at $5.2 \mathrm{GPa}$ that increases the O...O distance to $2.602 \AA$.

The distribution of the amide dimers in the CSD are shown in Fig 6 compared with the values observed in our study. The mean distance of the amide dimer in the CSD is $2.931 \AA$ with our model showing a dimer distance of 2.8855(19) A. As a comparator, pure saccharin has a dimer contact of $2.818 \AA$. On compression to $5.06 \mathrm{GPa}$ the hydrogen bond reduces by $8 \%$ to 2.655(10) $\AA$ again approaching the lowest values observed in the CSD. By restricting the CSD to show structures that have been studied under compression conditions (multiple datasets over a wide pressure range), there are only thirty refcodes observed pertaining to five molecules. Two polymorphs of 2oxopyrrolidineacetamide (piracetam),64 2-hydroxybenzamide (salicylamide) ${ }^{59}$ and a compression of two polymorphs of antidiabetic, tolazamide. ${ }^{65}$
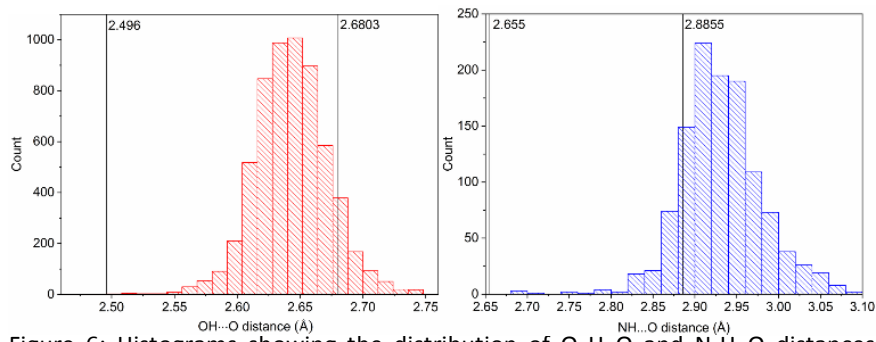

Figure 6: Histograms showing the distribution of $\mathrm{O}-\mathrm{H} \cdots \mathrm{NH}$... andistance $(\mathrm{A}) \mathrm{N}-\mathrm{H} \cdots \mathrm{O}$ distances reported in the CSD with markers for the distances reported in the compression
of INSA at ambient and the highest pressure dataset refined.

Figure 7 shows the changes in the hydrogen bonding in INSA compared with the dimer distances from other pressure studies of amides. The relative compression in each of these systems is comparable with a suggestion that the dimers in INSA and tolazamide are compressed a little faster. The reason behind this slight change in behaviour is potentially a combination of both the packing of the compounds and respective positioning of the hydrogen bonds in the structure. Both amide dimer contacts for piracetam ${ }^{64}$ and salicylamide ${ }^{59}$ are involved in a second hydrogen bonding interaction in an opposing direction within the structure which will aid in stabilising the amide dimer against shortening as a result of compression. Piracetam dimers form a hydrogen bond to the carbonyl of another neighbouring dimer unit in each direction, stabilising the central dimer. Piracetam undergoes a subtle phase transition after $0.45 \mathrm{GPa}$ 
from Form II to Form $\mathrm{V}$ which is the reason for the change in compression behaviour but the hydrogen bonding remains the same. The driving force for this transition is the reduction of voids in the structure. Salicylamide packs to form a channel of hydrogen bonding enabled by a screw axes. Each molecule in the dimer bonds perpendicularly bonds to another molecule, forming channels of hydrogen bonding. Piracetam and salicylamide also possess a layered structure where the hydrogen bonding is situated within the layer. The principal axis of strain was calculated to be approximately perpendicular to these layers in each case signifying that the main compression of the system comes from reducing the spacing between them hence the hydrogen bonding (in the layer) will be less impacted.

Tolazamide $^{65}$ polymorph II shows a similar rate of compression to INSA. The tolazamide structure exists as hydrogen-bonded dimers in layers with $\pi-\pi$ interactions between the layers. Like INSA, the amide dimer contact is not involved in any other hydrogen bonding therefore may be more susceptible to compression. Comparatively, the dimer observed in INSA is much closer at all pressure points than previous high pressure studies of piracetam, salicylamide, and tolazamide but is close to the average distance as observed in the database. The dimer in saccharin itself lies at $\sim 2.80 \AA$ depending on the temperature of the study. The other compounds under study possess longer $\mathrm{NH}$...O hydrogen bonding parameters at ambient pressure suggesting that the hydrogen bond interaction is lengthened to accommodate the packing of the molecules to a greater extent than in INSA. The packing indexes for each compound were calculated in Platon ${ }^{66}$ and found to be $73.0 \%$, $71.0 \%, 70.5 \%, 68.3 \%$ and $68.2 \%$ for piracetam, INSA, saccharin, tolazamide and salicylamide, respectively. Piracetam packs more efficiently than the rest but the nitrogen of the amide dimer is involved in another $\mathrm{N}-\mathrm{H} \cdots \mathrm{O}$ amide contact using the second hydrogen of the amide in the opposite direction which may serve to weaken and lengthen the dimer $h$-bond. Tolazamide and salicylamide have comparable lower packing indexes. Tolazamide is a relatively bulky structure that is more than double the molecular mass $(311.4 \mathrm{~g} / \mathrm{mol})$ of salicylamide $(137.1 \mathrm{~g} / \mathrm{mol})$. In the compression comparison Tolazamide was the only other structure with an amide dimer not involved in other hydrogen bonding that may allow a faster rate of compression. At ambient pressure, however, sterics will affect the packing of this structure inducing the $105^{\circ}$ angle at the sulphonamide group. In contrast, the low packing index for salicylamide is not due to sterics but as a result of the channelled hydrogen bonding and layered structure. INSA and saccharin itself possess an amide dimer that is free from other hydrogen bonding interactions. The pure substance is also a layered structure with a layer of hydrogen bonding with slip planes either side. The hydrogen bonding is supported by the close packing of the rings within the layer providing a stable environment for a short dimer contact.

A potential reason for the saccharin dimer being slightly longer in INSA is that the packing of the solid in INSA will be dominated by the packing of the indomethacin molecules due to its size. The saccharin dimer is in a pocket bookended by two indomethacin molecules. The saccharin dimer is not bound by the close packing of similar molecules (in the case of the pure compound) hence may be lying at an ideal $\mathrm{H}$-bonding distance for a 'gas-phase' dimer within this pocket. To support this hypothesis, dimer calculations were performed on the saccharin dimer at various set distances to probe the potential energy well. Figure $7 \mathrm{~b}$ shows the plot of the dimer energy versus distance for the saccharin dimer. Hydrogen-bonding values for the pure compound (SCCHRNO6) and the cocrystal (INSA) are indicated. As can be observed the potential energy well is rather shallow and the $\mathrm{h}$-bonding distances for both environments sit in the minimum. There is no energy penalty over the small separation distance hence we believe that the packing of the molecules in the immediate environment permits the saccharin dimer in the co-crystal to lie at a further distance. The low energy penalty for compressing this h-bond will facilitate its compression.

\section{Conclusion}

In this paper we have investigated the co-crystal of indomethacin and saccharin and its intermolecular interactions. The investigation of the continuous compression of the cocrystal showed no phase transition however there were some interesting points to note from the exploration of the structure on compression. Surprisingly, there are few compression studies involving acid or amide dimers in the CSD. A comparative study between INSA and other compression studies in the database with an amide dimer motif revealed that the amide bond compression was dependant on whether the amide was involved in further hydrogen bonding through the second amide hydrogen. The compression rate was slower for systems involving amide dimers with a second hydrogen bonding interaction but this is likely due to the extension of the hydrogen-bonding to form layered structures where the compression is maximised between layers rather than in the layer hence lower compression of the dimer. In comparison, INSA possesses a shorter amide dimer contact length and has a fast rate of compression that can be linked to the shallow potential energy well of the saccharin amide dimer. In terms of application to pharmaceutical development, this study provides a detailed understanding of how the co-crystal compresses and where the principal axes of strain lie in relation to the molecular species and intermolecular interactions. These observations are invaluable for the modelling of tabletting processes. How molecular species behave on compression can be utilised to improve models such that they may become predictive. In the future, by evaluating this relationship and encoding it, one may be able to design solids through crystal engineering to be more or less compressible in a particular direction thereby tuning the physical properties of the material to suit pharmaceutical processing. 
a)

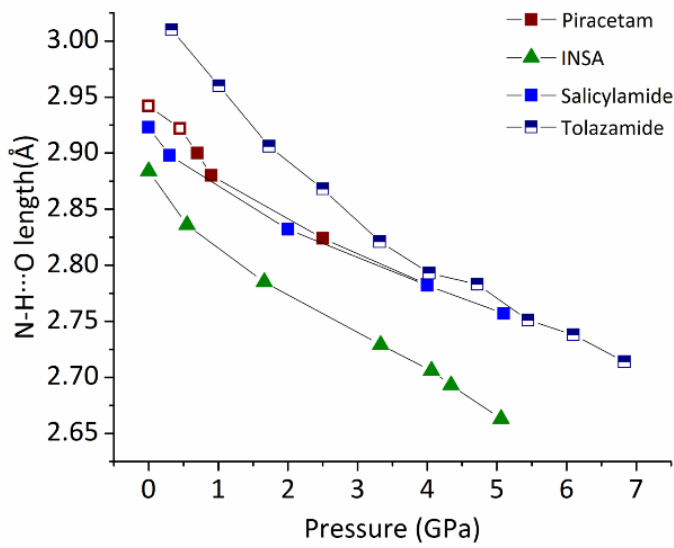

b)

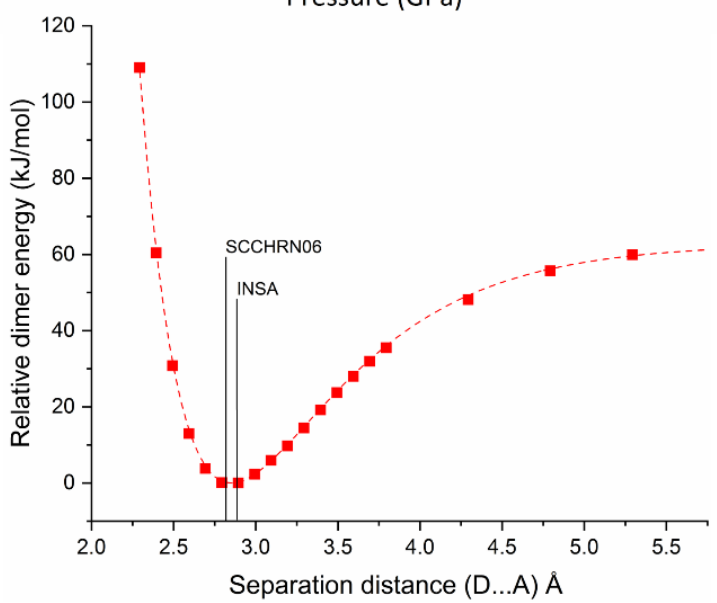

Figure 7: a) Plots showing how the length of piracetam (form II: half symbol \& form $\mathrm{V}$ : filled symbol), INSA, tolazamide, and salicylamide's $\mathrm{N}-\mathrm{H} \cdots \mathrm{O}$ amide dime respond to pressure. b) The saccharin dimer single point energy at increasing hydrogen bonding distance (N...O distance). The calculation was performed at De (diG of (equilibrium distance) $=2.852(3) \AA$

\section{Conflicts of interest}

"There are no conflicts to declare".

\section{Acknowledgements}

The authors gratefully acknowledge the financial support from the EPSRC for funding (EP/N015401/1, EP/R032858/1). We would like to thank the International Strategic Partnership between University of Strathclyde and Nanyang Technological University Singapore for funding this work. GWH is funded by Cancer Research UK (C149/A20496). The authors would like to acknowledge that this work was carried out in the CMAC National Facility supported by UKRPIF (UK Research Partnership Fund) award from the Higher Education Funding Council for England (HEFCE) (Grant Ref: HH13054). All data underpinning this publication are openly available from the University of Strathclyde KnowledgeBase at https://doi.org/10.15129/a65c26b6-3b97-4bd8-8d4a- efcb7c91f9b8 (to be validated once article is accepted). CCDC deposition numbers are CCDC 1902709-1902715.

\section{Notes and references}

1 K. B. Landenberger and A. J. Matzger, Cryst. Growth Des., 2010, 10, 5341-5347.

2 Z. Yang, H. Li, X. Zhou, C. Zhang, H. Huang, J. Li and F. Nie, Cryst. Growth Des., 2012, 12, 5155-5158.

3 E. C. Subbarao, G. Shirane and F. Jona, Acta Crystallogr., 1960, 13, 226-231.

4

G. Bolla, H. Dong, Y. Zhen, Z. Wang and W. Hu, Sci. China Mater., 2016, 59, 523-530.

5 S. Karki, T. Friić, L. Fábián and W. Jones, CrystEngComm, 2010, 12, 4038-4041. S. S. Kumar, R. Thakuria and A. Nangia, CrystEngComm, 2014, 4722-4731.

7

8 J. W. Steed, Trends Pharmacol. Sci., 2013, 34, 185-193. N. Schultheiss and A. Newman, Cryst. Growth Des., 2009, 9, 2950-2967.

D. I. A. Millar, H. E. Maynard-Casely, D. R. Allan, A. S. Cumming, A. R. Lennie, A. J. Mackay, I. D. H. Oswald, C. C. Tang and C. R. Pulham, CrystEngComm, 2012, 14, 3742. G. R. Krishna, L. Shi, P. P. Bag, C. C. Sun and C. M. Reddy, Cryst. Growth Des., 2015, 15, 1827-1832.

S. Ghosh and C. M. Reddy, Angew. Chemie - Int. Ed., 2012, 51, 10319-10323.

A. Mukherjee and G. R. Desiraju, IUCrJ, 2014, 1, 49-60. C. M. Reddy, R. C. Gundakaram, S. Basavoju, M. T. Kirchner, K. A. Padmanabhan and G. R. Desiraju, Chem. Commun., 2005, 3945-3947.

A. J. Cruz-Cabeza, S. M. Reutzel-Edens and J. Bernstein, Chem. Soc. Rev., 2015, 44, 8619-8635.

Ö. Almarsson and M. J. Zaworotko, Chem. Commun., 2004, 1889-1896.

K. Nidhi, S. Indrajeet, M. Khushboo, K. Gauri and D. J. Sen, J. Pharm. Sci., 2006, 95, 2145-2157.

L. S. Reddy, N. J. Babu and A. Nangia, Chem. Commun., 2006, 1369-1371.

E. V. Boldyreva, T. P. Shakhtshneider, H. Ahsbahs, H. Sowa and H. Uchtmann, J. Therm. Anal. Calorim., 2002, 68, 437452.

E. Eikeland, M. K. Thomsen, S. R. Madsen, J. Overgaard, M. A. Spackman and B. B. Iversen, Chem. - A Eur. J., 2016, 22, 4061-4069.

I. D. H. Oswald, A. R. Lennie, C. R. Pulham and K. Shankland, CrystEngComm, 2010, 12, 2533.

K. Dziubek, M. Citroni, S. Fanetti, A. B. Cairns and R. Bini, J. Phys. Chem. C, 2017, 121, 2380-2387.

K. Roszak, A. Katrusiek and A. Katrusiak, Cryst. Growth Des., 2016, 16, 3947-3953.

F. P. A. Fabbiani, D. R. Allan, S. Parsons and C. R. Pulham, CrystEngComm, 2005, 7, 179.

K. Wang, J. Liu, K. Yang, B. Liu and B. Zou, J. Phys. Chem. C, 2014, 118, 8122-8127.

E. V. Boldyreva, Zeitschrift fur Krist., 2014, 229, 236-245. 
E. Patyk, J. Skumiel, M. Podsiadlo and A. Katrusiak, Angew. Chemie-International Ed., 2012, 51, 2146-2150. C. L. Bull, G. Flowitt-Hill, S. de Gironcoli, E. Küçükbenli, S. Parsons, C. Huy Pham, H. Y. Playford and M. G. Tucker, 2017, 4, 569-574. N. P. Funnell, W. G. Marshall and S. Parsons, CrystEngComm, 2011, 13, 5841-5848. B. A. Zakharov and E. V. Boldyreva, Acta Crystallogr. Sect. B Struct. Sci. Cryst. Eng. Mater., 2013, 69, 271-280. K. Wang, D. Duan, R. Wang, D. Liu, L. Tang, T. Cui, B. Liu, Q. Cui, J. Liu, B. Zou and G. Zou, J. Phys. Chem. B, 2009, 113, 14719-14724. E. Patyk and A. Katrusiak, Chem. Sci., 2015, 6, 1991-1995. C. R. Groom, I. J. Bruno, M. P. Lightfoot and S. C. Ward, Acta Crystallogr. Sect. B Struct. Sci. Cryst. Eng. Mater., 2016, B72, 171-179.

Pharm. Sci., 2006, 95, 689-700. F. P. A. Fabbiani, G. Buth, D. C. Levendis and A. J. CruzCabeza, Chem. Commun. (Camb)., 2014, 50, 1817-9. S. Basavoju, D. Boström and S. P. Velaga, Pharm. Res. 2008, 25, 530-541.

M. Jung, J. Kim, M. Kim, A. Alhalaweh, W. Cho, S. Hwang and S. P. Velaga, J. Pharm. Pharamcology, 2010, 62, 15601568.

G. J. Piermarini, S. Block, J. D. Barnett and R. a. Forman, J. Appl. Phys., 1975, 46, 2774-2780. Bruker AXS Inc., APEX II, 2013, Bruker AXS Inc., Madison, Wisconsin, USA. G. M. Sheldrick, SADABS, Programs Scaling Absorpt. Correct. Area Detect. Data, 2008, University of Göttingen: Göttingen, Germany. and H. Puschmann, J. Appl. Crystallogr., 2009, 42, 339-341. A. Dawson, D. R. Allan, S. Parsons and M. Ruf, J. Appl. Crystallogr., 2004, 37, 410-416. S. Parsons, 2004.

C. F. Macrae, I. J. Bruno, J. A. Chisholm, P. R. Edgington, P. McCabe, E. Pidcock, L. Rodriguez-Monge, R. Taylor, J. van de Streek and P. A. Wood, J. Appl. Crystallogr., 2008, 41, 466-470.

D. E. Curtis, T. Vandermeersch, G. R. Hutchison, D. C. Lonie, E. Zurek and M. D. Hanwell, J. Cheminform., 2012, 4, 17. M. J. Frisch, G. W. Trucks, H. B. Schlegel, G. E. Scuseria, M. A. Robb, J. R. Cheeseman, G. Scalmani, V. Barone, B. Mennucci, G. A. Petersson, H. Nakatsuji, M. Caricato, X. Li, H. P. Hratchian, A. F. Izmaylov, J. Bloino, G. Zheng, J. L. Sonnenberg, M. Hada, M. Ehara, K. Toyota, R. Fukuda, J. Hasegawa, M. Ishida, T. Nakajima, Y. Honda, O. Kitao, H. Nakai, T. Vreven, J. A. Montgomery, J. E. Peralta, F. Ogliaro, M. Bearpark, J. J. Heyd, E. Brothers, K. N. Kudin, V. N. Staroverov, R. Kobayashi, J. Normand, K. Raghavachari, A. Rendell, J. C. Burant, S. S. Iyengar, J. Tomasi, M. Cossi, N. Rega, J. M. Millam, M. Klene, J. E. Knox, J. B. Cross, V. Bakken, C. Adamo, J. Jaramillo, R. Gomperts, R. E. Stratmann, O. Yazyev, A. J. Austin, R. Cammi, C. Pomelli, J. W. Ochterski, R. L. Martin, K. Morokuma, V. G. Zakrzewski,
G. A. Voth, P. Salvador, J. J. Dannenberg, S. Dapprich, A. D. Daniels, Farkas, J. B. Foresman, J. V Ortiz, J. Cioslowski and D. J. Fox, 2009.

46 A. Gavezzotti, New J. Chem., 2011, 35, 1360-1368.

47 A. Gavezzotti, J. Phys. Chem. B, 2003, 107, 2344-2353.

48 A. Gavezzotti, Mol. Phys., 2008, 106, 1473-1485.

49 L. Maschio, B. Civalleri, P. Ugliengo and A. Gavezzotti, J. Phys. Chem., 2011, 115, 11179-11186.

50 J. Gonzalez-Platas, M. Alvaro, F. Nestola and R. Angel, J. Appl. Crystallogr., 2016, 49, 1377-1382.

51 M. J. Cliffe and A. L. Goodwin, J. Appl. Cryst, 2012, 45, 1321-1329.

F. A. Momany, R. F. McGuire, A. W. Burgess and H. A. Scheraga, J. Phys. Chem., 2005, 79, 2361-2381. L. Pauling, R. B. Corey and H. R. Branson, Proc. Natl. Acad. Sci., 1951, 37, 205-211. J. Bernstein, R. E. Davis, L. Shimoni and N. -L Chang, Angew. Chemie Int. Ed. English, 1995, 34, 1555-1573. S. A. Moggach, S. Parsons and P. A. Wood, Crystallogr. Rev., 2008, 14, 143-184.

C. H. Görbitz, Crystallogr. Rev., 2015, 21, 160-212. P. Lozano-Casal, D. R. Allan and S. Parsons, Acta Crystallogr. Sect. B Struct. Sci., 2008, 64, 466-475. N. A. Tumanov and E. V Boldyreva, Acta Crystallogr. Sect. B-Structural Sci., 2012, 68, 412-423.

R. D. L. Johnstone, A. R. Lennie, S. F. Parker, S. Parsons, E. Pidcock, P. R. Richardson, J. E. Warren and P. A. Wood, CrystEngComm, 2010, 12, 983-1316.

60 K. Ostrowska, M. Kropidowska and A. Katrusiak, Cryst. Growth Des., 2015, 15, 1512-1517.

A. Delori, I. B. Hutchison, C. L. Bull, N. P. Funnell, A. J. Urquhart and I. D. H. Oswald, Cryst. Growth Des., 2018, 18, 1425-1431.

T. Galica, J. Bakowicz, K. Konieczny and I. Turowska-Tyrk, Cryst. Growth Des., 2018, 18, 1636-1644. T. Galica, J. Bąkowicz, K. Konieczny and I. Turowska-Tyrk, CrystEngComm, 2016, 18, 8871-8879.

F. P. A. Fabbiani, D. R. Allan, W. I. F. David, A. J. Davidson, A. R. Lennie, S. Parsons, C. R. Pulham and J. E. Warren, Cryst. Growth Des., 2007, 7, 1115-1124.

A. Y. Fedorov, D. A. Rychkov, E. A. Losev, B. A. Zakharov, J. Stare and E. V. Boldyreva, CrystEngComm, 2017, 19, 22432252.

A. L. Spek, Acta Crystallogr. Sect. D Biol. Crystallogr., 2009, 65, 148-155. 